\title{
A REGULAR SEMI-METRIC SPACE FOR WHICH THERE IS NO SEMI-METRIC UNDER WHICH ALL SPHERES ARE OPEN
}

\author{
ROBERT W. HEATH
}

A topological space $S$ is said to be a semi-metric space provided that there is a distance function (or "semi-metric") $d$ defined for $S$ such that, if each of $x$ and $y$ is a point of $S$, then (1) $d(x, y)=d(y, x)$, (2) $d(x, y) \geqq 0$ and $d(x, y)=0$ only in case $x=y$, and (3) the topology of $S$ is invariant with respect to the distance function $d-$ i.e., if $p$ is a limit point of a subset $M$ of $S$, then $p$ is a distance limit point of $M$ and conversely. If $x \in S$ and $c>0,\{y: y \in S$ and $d(x, y)<c\}$ is a $c$-neighborhood (or "c-sphere") of the point $x$.

For the purpose of constructing semi-metric spaces the following characterization from [1] is useful.

A necessary and sufficient condition that the topological space $S$ be semi-metric is that there exist a collection of open sets, $\left\{g_{n}(x): x \in S, n=1,2,3, \cdots\right\}$, such that (1) for each $x \in S$, $\left\{g_{n}(x)\right\}_{n=1}^{\infty}$ is a nonincreasing sequence which forms a local base for the topology at $x$, and (2) if $y \in S$ and $x$ is a point sequence in $S$ such that, for each $m, y \in g_{m}\left(x_{m}\right)$, then $x$ converges to $y$.

A good source of information on some of the work that has been done on semi-metric spaces is to be found in [4]. As is pointed out there by F. B. Jones, a regular semi-metric space is not too far removed from a Moore space (every Moore space being a regular semimetric space) in that most of the theorems of Moore's book hold in a regular semi-metric space (with the addition of an appropriate completeness axiom, local connectedness, etc., as needed)-in fact a strongly complete regular semi-metric space is a Moore space [1].

In [4] Morton Brown noted several open questions including: "Does every semi-metric space have a semi-metric under which all spheres are open?" It is well known that every Moore space does have such a semi-metric; however, the general question is answered in the negative by the following theorem.

THEOREM. There exists a regular semi-metric space which is normal, paracompact, hereditarily separable, locally connected, and weakly complete, but for which there is no distance function with respect to which all neighborhoods (or "spheres") are open. 1960.

Presented to the Society, August, 30,1960; received by the editors October 1, 
Proof. The space consists of the points of the plane with a basis made up of (1) all open discs that either do not intersect the $x$-axis or are centered on rational points of the $x$-axis and (2) all "bow-tie regions" centered on irrational points of the $x$-axis-i.e., for each irrational point $x$ and each $c>0$, every set of the form $\{y:|x-y|+a(x, y)<c\}$, where $|x-y|$ is the Cartesian distance from $x$ to $y$ and $a(x, y)$ is the smallest non-negative angle (in radians) formed by the $x$-axis and a line containing $x$ and $y$.

That the space is semi-metric follows immediately using the characterization from [1] noted above. The other properties (except the last one) possessed by the space follow as in the proof of Theorem 3.2 of [2], this space being sufficiently similar to Example 3.1 of [2].

Finally, there is no distance function with respect to which all neighborhoods are open. For suppose that $d$ is any distance function for the space. Then there is a $c>0$ and a second category (relative to the topology of the $x$-axis) subset $M$ of the irrationals on the $x$-axis such that, if $x \in M$, then a $c$-neighborhood (with respect to $d$ ) about $x$ must be contained in a bow-tie region of radius 1 about $x$ $(\{y:|x-y|+a(x, y)<1\})$. Thus if $r$ is a rational point of the $x$-axis which is a limit point of $M$, and $p$ is a point with the same abscissa as $r(p \neq r)$ such that $d(p, r)<c / 2$, then $U_{c / 2}(p)$ contains $r$ but cannot contain more than a finite number of terms of any point sequence in $M$ that converges to $r$. There must be such a sequence; therefore $U_{c / 2}(p)$ is not open.

Note that the example can be altered (by defining the topology on all lines parallel to the $x$-axis and intersecting the $y$-axis in rational points in the same way as it is defined on the $x$-axis above) in such a way that the set of all points about which not all neighborhoods are open must be dense in the space.

\section{REFERENCES}

1. R. W. Heath, Arc-wise connectedness in semi-metric spaces, to appear.

2. L. F. McAuley, A relation between perfect separability, completeness, and normality in semi-metric spaces, Pacific J. Math. vol. 6 (1956) p. 317.

3. R. L. Moore, Foundations of point set theory, Amer. Math. Soc. Colloquium Publications, vol. 13, 1932.

4. Seminar on semi-metric spaces, Summary of Lectures and Seminars, Summer Institute on Set Theoretic Topology, Madison, Wisconsin, 1955.

University of Georgia 